\title{
Interpretation of borehole-inclinometer data: a general theory applied to a new instrument
}

\author{
Erik W. Blake and Garry K. C. Clarke \\ Department of Geophysics and Astronomy, University of British Columbia, Vancouver, \\ British Columbia V6T 1W5, Canada
}

\begin{abstract}
Borehole inclinometers are standard equipment for field glaciologists and are commonly used for investigating the flow law of ice and for measuring the spatial position of englacial and sub-surface sensors. The recent development, at the University of British Columbia (UBC), of a prototype inclinometer that employs a three-component fluxgate magentometer to obtain a compass bearing has stimulated our interest in borehole inclinometry. Following a review of various approaches to glacier inclinometry, we present a unified theory of data interpretation that can be applied to all inclinometers, discuss the application of the theory to the UBC inclinometer, and discuss the sensitivity of the theory to error in the data.
\end{abstract}

\section{INTRODUCTION}

Whenever a deep hole is drilled into the surface of the Earth, there is uncertainty about its trajectory. Asymmetries in the drill, layering in the material being drilled and shortcomings in drilling technique may cause the borehole to stray from its intended path (e.g. Walker, 1986). Inclinometry tools are designed to remove spatial uncertainty by mapping the deviation of the borehole. Typically, two pieces of information are collected at a series of stations, or locations, along the length of the hole: the tilt of the inclinometer from vertical and the azimuth of that tilt in a geographical coordinate system. Of the many techniques available for making these measurements, we present a brief overview of those that have been used for glaciological work. Sources for some of this inclinometry equipment are given.

In this paper, we attempt to formalize some aspects of inclinometry-data analysis and, at the same time, introduce a new inclinometry tool that was recently assembled at the University of British Columbia (UBC). We describe the calibration procedure for the UBC inclinometer, formulate a general theory for interpretation of inclinometer data and examine the sensitivity of this theory to error in the data.

\section{HISTORICAL OVERVIEW}

Inclinometry of glacier boreholes has long been associated with field investigations of the flow law for ice (Perutz, 1947, 1949, 1950; Gerrard and others, 1952; Sharp, 1953; Nye, 1952, 1953, 1957; Miller, 1958; Paterson and Savage, 1963; Savage and Paterson, 1963; Shreve and Sharp, 1970; Raymond, 1971b, c; Hooke, 1973; Garfield and Ueda, 1976; Paterson, 1983; Hooke and Hanson,
1986; Hooke and others, 1987). Many of these researchers used inclinometry data to infer the internal velocity field of the glacier and thereby deduce parameters of the flow law. Increasing interest in basal processes has created a new application for inclinometry, that of accurately determining the position of englacial and subglacial sensors. As an example, the measurement of subglacial resistivity (Brand and others, 1987) requires knowledge of the spatial position of current and voltage electrodes within adjacent boreholes.

\section{Basic dip and azimuth measurements}

A rudimentary fluid-level inclinometer can be built by partially filling a sealed glass vial with a solution of hydrofluoric acid. If the vial is kept at a fixed tilt angle for an appropriate time, the acid will etch the glass, leaving a record of the fluid surface. If several vials are positioned along the length of a borehole while the etching occurs, they provide a discrete record of the borehole tilt (e.g. Savage and Paterson, 1963, p. 4522). An exotic variation of the above approach substitutes a warm solution of gelatin ("Jello" is a suitable commercial product) for the acid; the vials are left in place until the gelatin hardens and tilt is preserved in the surface of the gelatin (Shreve and Sharp, 1970, p. 71). Such fluid levels have three major drawbacks: (1) The vials may freeze to the walls of the borehole. (2) The precision of the tilt measurements is poor. (3) The vials do not measure tilt azimuth, although, in the case of the gelatin level, a magnetic compass needle suspended within the gelatin can provide a magnetic bearing.

"Floating-compass" inclinometers improve on fluidlevel instruments by providing azimuth information, but they are laborious to use. Sperry-Sun Corporation (Houston, Texas) and Parsons Survey Company (South 
Gate, California) manufacture floating-compass inclinometers that employ a miniature camera. The camera in the Sperry-Sun instrument is focused on a weighted spherical magnetic compass ball that has azimuth and tilt markings, much like lines of latitude and longitude. At predetermined time intervals, the camera takes a photograph of the illuminated compass ball; it is left to the operator to ensure that the instrument is at the proper position along the borehole at the time each picture is taken. The camera in the Parsons instrument photographs an illuminated plumb bob against a compass graticule. The single-frame version of this instrument must be brought to the surface after each picture has been taken (e.g. Shreve, 1961; Savage and Paterson, 1963), but an improved multi-frame version uses electrical signals from the surface to advance the film (Garfield and Ueda, 1976). With both the Sperry-Sun and Parsons instruments, azimuth and tilt readings are transcribed from the developed film images, an obvious impediment to in-field data acquisition.

Clamping the compass so that it can be examined once the inclinometer is brought to the surface is an alternative to film recording of compass position. The inclinometer built by Pajari Instruments Ltd (Orillia, Ontario) uses a gimbal-mounted horizontal compass needle. After a predetermined interval of time has passed, a locking mechanism arrests the gimbal rings and the compass. The operator sets the timer, lowers the instrument to the desired location and waits for the timer to lock the compass. The inclinometer is then brought to the surface, the gimbal mount is removed from the pressure casing, and azimuth and tilt are read from graduated scales in the unit. This laborious procedure is repeated for each station within the borehole. Koerner (personal communication, 1987) has used this instrument on the Agassiz Ice Cap.

\section{ELECTRONIC INCLINOMETRY}

In recent years, advances in electronic technology have enabled the development of inclinometry systems that transmit position information directly to the surface. Compared with earlier techniques, the time and labour savings of electronic inclinometry are appreciable; with appropriate equipment in place at the borehole site, the trajectory of the borehole can even be computed and displayed as the data are collected.

\section{Measuring tilt}

Electronic tilt transducers allow precise and repeated measurements of tilt to be made from a glacier surface. The Fredericks Company and Applied Geomechanics Inc. (Santa Cruz, California) manufacture electrolytic tilt transducers that are suitable for borehole inclinometry. General Oceanics (Miami, Florida) and Slope Indicator Company (Seattle, Washington) manufacture forcebalance tilt transducers.

\section{Measuring azimuth}

To reconstruct the borehole trajectory, each set of tilt measurements must be combined with a measurement of instrument azimuth. Rigidly coupled inclinometers, tracked inclinometers, magnetically oriented inclinometers and gyroscopically oriented inclinometers are the four design categories known to us.

\section{External azimuth control}

The orientation of a rigidly coupled inclinometer is controlled by a rod leading from the surface. This method is suitable for shallow holes but, as the distance between the instrument and the operator increases, azimuth control deteriorates and logistical problems grow (Gerrard and others, 1952).

Tracked inclinometers require that a special grooved casing be installed in the borehole before inclinometry begins. Spring-loaded wheels or pins keep the inclinometer aligned with the grooves in the casing as the instrument moves through the hole. For shallow holes, twisting of the casing is assumed to be negligible; it follows that the azimuth of the inclinometer is constant and equal to the azimuth of the casing at the top of the hole. For deeper holes, a torsion tool can be used to measure the twist in the casing. This tool consists of two sections, each of which has a set of tracking wheels, that are connected by a sensitive torsion transducer. Any twisting of the casing is recorded by the transducer as the tool is moved through the hole. If a torsion tool is not available, the presence or absence of casing twist must be inferred from the data. Although the effort and expense of installing an inclinometry casing is an inconvenience, the casing provides a uniform borehole geometry for the inclinometer, serves to smooth out small perturbations in the borehole trajectory, and allows the operator to cancel any offset error in the instrument by executing precisely reversed runs through the hole; great accuracy can be achieved. Cased holes are also advantageous in long-term deformation studies because they are not prone to closure from creep and freezing. The biaxial inclinometer manufactured by Slope Indicator Company (Seattle, Washington) is an excellent example of a tracked inclinometer. Hooke and Hanson (1986) and Hooke and others (1987) have made precise measurements of glacier-ice deformation using this instrument, but we are certain that they have cursed the need for a casing.

\section{Internal azimuth control}

Gyroscopically and magnetically oriented inclinometers do not require a casing, hence their appeal for glacier work. A gyroscopically oriented inclinometer was built by the research team that made the historic measurements of deformation within Jungfraufirn (Gerrard and others, 1952). Their paper contains a brief description of this instrument, but we have been unable to locate the detailed instrumentation paper to which they refer (Broad, Jason and Perutz. F. Sci. Instrum., 1952, designated "in press") and suspect it remains unpublished.

Inclinometers that use a magnetic compass to determine azimuth and that report the azimuth electrically are a vast improvement over the Sperry-Sun, Parsons and Pajari designs; the output from the tilt sensors and the compass can be recorded while the inclinometer is on station. The primary disadvantage of magnetic orientation is that the compass is sensitive to geomagnetic 
disturbances, as well as magnetic field variations caused by remnant magnetization and magnetic susceptibility in nearby materials. For ice, which has no magnetic signature, only geomagnetic disturbances are of consequence. Glaciologists have used several designs for magnetically oriented inclinometers. Raymond (1971a) described an instrument that was used to measure the internal velocity structure of Athabasca Glacier (Raymond, 1971c). P. Taylor (Hydro-Tech, Seattle, Washington) has built several magnetically oriented inclinometers specifically for use in glaciers. These instruments employ two orthogonal tilt sensors and a gimbal-mounted compass that produces a voltage signal proportional to the magnetic bearing.

Further improvements in reliability can be gained by using a non-mechanical compass, such as a magnetometer. Levanto (1959) used a down-hole three-component fluxgate magnetometer to measure the in situ magnetic susceptibility of rock. The instrument did contain a tilt sensor, but the sensor was used to maintain the attitude of the magnetometer rather than measure inclination. With minor modification, this instrument would have served well as an inclinometer.

\section{THE UBC INCLINOMETER}

In the spring of 1989, the glaciology group at the University of British Columbia, in collaboration with Slope Indicator Canada Limited (Richmond, British Columbia) and Narod Geophysics (Vancouver, British Columbia), developed a prototype inclinometer. This instrument is similar to the Hydro-Tech instrument in that it uses the Earth's magnetic field as an azimuth reference, but the field-sensing device is a fluxgate magnetometer rather than a mechanical compass. Two force-balance tilt transducers and the magnetometer are enclosed within a non-magnetic stainless-steel pressure tube (see Fig. 1). Brass centralizing springs help keep the

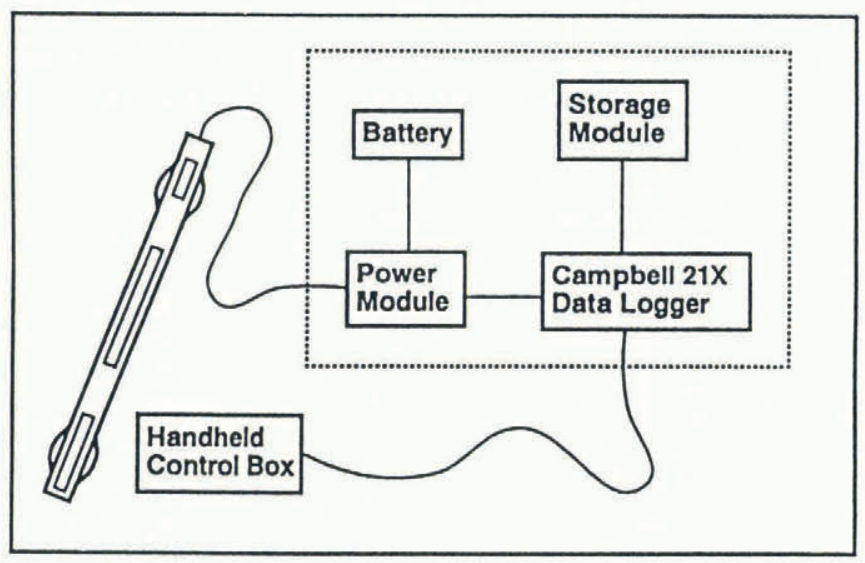

Fig. 1. A block diagram of the peripheral devices attached to the UBC inclinometer (inclinometer not drawn to scale). The power module provides power to the instrument from a small 6 Ah sealed lead-acid battery. Analogue signals are fed to the data logger which, on command from the handheld control box, records data from the tilt sensors and magnetometer into the storage module. The operator keys in depth information on the control box. inclinometer centred in the borehole and prevent the instrument from spinning when on station. The tube is $1.5 \mathrm{~m}$ long and has an outside diameter of $2.54 \mathrm{~cm}$ (1.00 in). The tilt transducers are Slope Indicator devices, identical to those used in their tracked inclinometers. The magnetometer is a Narod Geophysics miniature ring-core fluxgate magnetometer. (Wyckoff (1948) gave an overview of the principals of operation for a fluxgate magnetometer; more detailed treatments related to this magnetometer can be found in: United States Patent 3800213 (26 March 1974), UK Patent 2044460 (16 October 1979), Russell and others (1983), Narod and Russell (1984) and Narod and others (1985)). The sensing element of this three-component magnetometer is a $12.7 \mathrm{~mm}(0.50 \mathrm{in})$ cube. As the tilt sensors contain permanent magnets, the magnetometer is mounted as far from the tilt sensors as possible. Ideally, the magnetometer would be placed at the bottom of the instrument, far from any secondary magnetic fields, but the tilt sensors completely fill the interior of the pressure tube so that no wires can pass by them; thus the tilt sensors must be positioned at the bottom and the magnetometer at the top. The space between these sensors is occupied by the electronics package. The instrument cable attaches to the top of the instrument. This cable carries power to the instrument and analogue data to the surface, and has a steel strength member. The magnetic bias created by the steel cable and the electric currents flowing in the cable is compensated by the magnetometer calibration.

Data are collected by a data logger mounted inside a weather-tight plastic case. For each hole, the inclinometer is stepped down and up at $1 \mathrm{~m}$ intervals, resulting in a two-fold redundancy in the measurements. The depth of the inclinometer at each station, as marked on the cable, is coded manually on a digital potentiometer that is mounted in a hand-held control box. After the operator closes a momentary switch, indicating that the inclinometer is on station, the data logger records the time, the digital values of the tilt and magnetometer signals, and the voltage on the potentiometer. The data logger copies the data into the storage module. At the end of the day, the storage module is carried to the field camp where the data are transferred to a computer for analysis.

This inclinometer system is very efficient. A single operator can make two inclinometry passes through a $70 \mathrm{~m}$ hole at $1 \mathrm{~m}$ depth intervals in $25 \mathrm{~min}$.

\section{COORDINATE SYSTEMS}

Three right-handed Cartesian coordinate systems are required to process data from a tracked or gyroscopically oriented inclinometer. Two additional coordinate systems associated with the Earth's magnetic field and the magnetometer are required to process data from a magnetically oriented inclinometer (Fig. 2). The coordinate system introduced by Nye (1957), and used in several subsequent papers by Nye and others, is aligned with the flow direction of the glacier. This is natural for studies of internal deformation but, because our primary concern is locating sensors placed within boreholes, we choose a system related to map coordinates. 


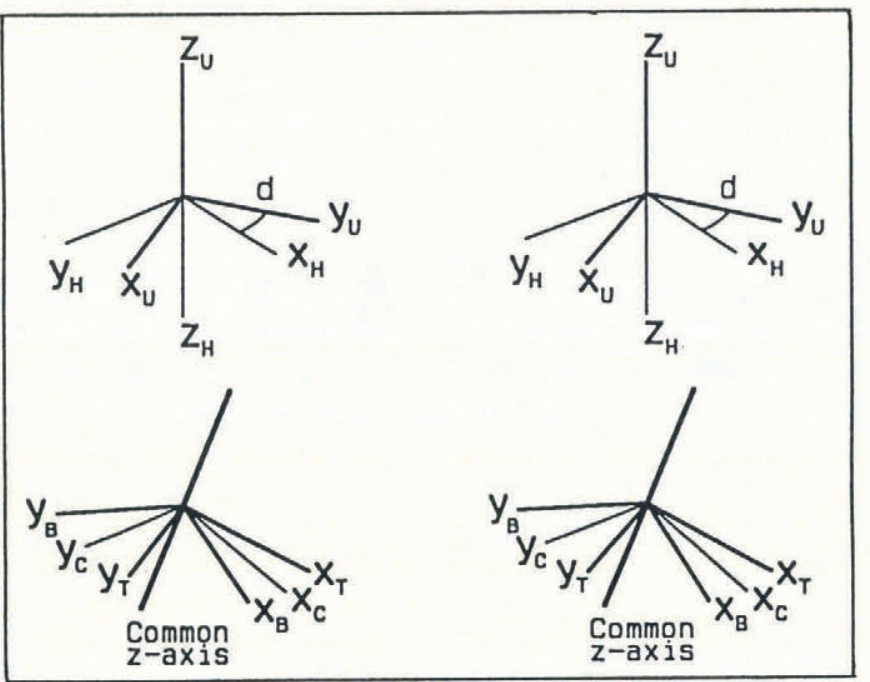

Fig. 2. The five righthanded inclinometer coordinate systems displayed as a stereo pair. Top: the geographical coordinate system $U$ and the geomagnetic coordinate system $H$. The angle " $d$ " between the grid north axis $y_{\mathrm{U}}$ and the magnetic north axis $x_{\mathrm{H}}$ is the magnetic declination. Bottom: the tilt sensor $(T)$, case $(C)$, and magnetometer (B) coordinate systems share a common $z$ axis pointing downward along the body of the inclinometer.

The geographical coordinate system $U$ is tied to the Universal Transverse Mercator (UTM) coordinate system used on Canadian topographic maps. The $x_{\mathrm{U}}, y_{\mathrm{U}}$ and $z_{\mathrm{U}}$ axes point east, north and upward, respectively. The geomagnetic coordinate system (labelled $\mathrm{H}$ after the standard designation for magnetic field) is tied to the local declination of the Earth's magnetic field. The $z_{\mathrm{H}}$ axis is positive downward and the $x_{H}$ axis points along the magnetic declination. This definition follows the international convention for geomagnetic coordinate systems and results in positive $z_{\mathrm{H}}$ readings in the Northern Magnetic Hemisphere. The remaining three coordinate systems have $z$-axes that point downward along the axis of the inclinometer. The tiltsensor coordinate system $\mathrm{T}$ has its $x_{\mathrm{T}}$ and $y_{\mathrm{T}}$ axes aligned with the sensitive axes of the tilt sensors. The case coordinate system $\mathrm{C}$ has the $x_{\mathrm{C}}$ axis aligned with some feature on the inclinometer. In the case of a tracked inclinometer, this would be the alignment mechanism. The UBC instrument has its $x_{\mathrm{C}}$ axis aligned with a machined facet on the inclinometer case (the use of this facet is discussed below). The magnetometer coordinate system (labelled $\mathrm{B}$ after the standard designation for magnetic flux density) represents the three axes of sensitivity of the fluxgate magnetometer. For the UBC inclinometer, the three instrument-based coordinate systems are distinct, and the rotational angles between the $x_{\mathrm{T}}, x_{\mathrm{C}}$ and $x_{\mathrm{B}}$ axes must be known in order to process data. For other instruments, these three systems may or may not coincide.

\section{DATA ANALYSIS}

The analysis of inclinometer data proceeds in three distinct steps: (1) The instrument calibrations are used to compute the two tilt angles and the azimuth at each station. (2) The tilt and azimuth values are transformed into vectors representing the orientation of the inclinometer at each station. (3) An inversion scheme computes the continuous trajectory of the borehole based on the discrete set of orientations.

In the following two sections, we discuss in detail the calibration and transformation procedures for the UBC inclinometer. Although these procedures vary from instrument to instrument, all share the common goal of determining the vertical unit vector $\hat{\mathbf{n}}$ and the orientation unit vector $\hat{\mathbf{m}}$ in an inclinometer coordinate system (Fig. 3). For a magnetically oriented inclinometer, $\hat{\mathbf{m}}$ represents the Earth's magnetic field vector and, for a tracked inclinometer, $\hat{\mathbf{m}}$ represents the orientation of the track grooves. The UBC inclinometer yields the true magnetic vector, whereas an instrument that uses a gimbal-mounted compass yields the magnetic vector projected on to the gravitationally horizontal plane.

\section{Calibration}

The tilt sensors are calibrated using a calibration frame that allows positioning of the inclinometer at precise tilt angles (to within $1 \mathrm{~min}$ of arc) throughout its operating range of $\pm 30^{\circ}$ of tilt from vertical. Repeated calibration of the instrument between field seasons indicates negligible drift in the calibration. The output voltage from the tilt sensors is proportional to the sine of the tilt angle from vertical. In the $\mathrm{T}$ system, the components of $\hat{\mathbf{n}}$ are given by

$$
\left\{\begin{array}{l}
n_{x}=\sin I_{x} \\
n_{y}=\sin I_{y} \\
n_{z}=-\left[1-\left(n_{x}^{2}+n_{y}^{2}\right)\right]^{\frac{1}{2}}
\end{array}\right\}
$$

where $I_{x}$ and $I_{y}$ are the two tilt angles.

The calculation of the orientation vector $\hat{\mathbf{m}}$ would be trivial if the three axes of the magnetometer were to have zero offset and equal sensitivity but, in practice, the design of the magnetometer sensor makes it very difficult to realize these characteristics, particularly with regard to

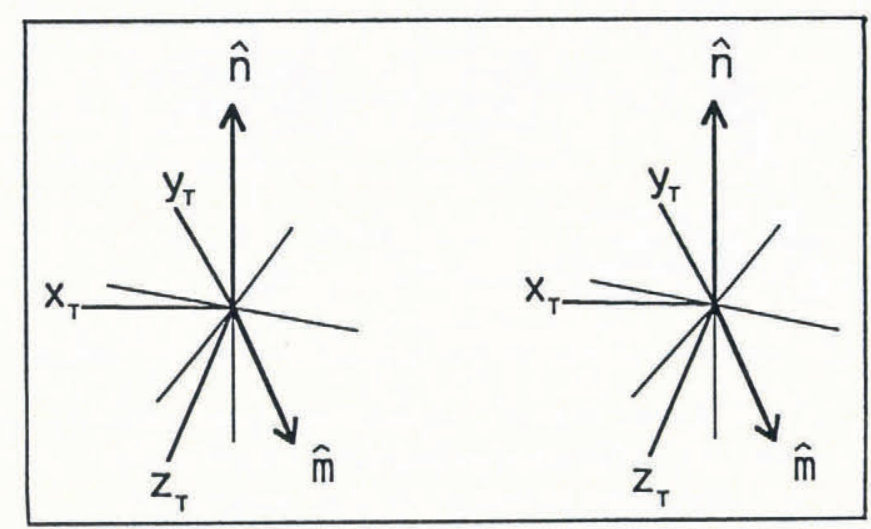

Fig. 3. A stereogram showing the two unit vectors measured by an inclinometer and the tilt coordinate system $T$ in which they are resolved. The tilt sensors yield $\hat{\mathbf{n}}$ and the orientation apparatus yields $\hat{\mathbf{m}}$. 
voltage offsets. A calibration is required. If a series of measurements of the Earth's magnetic field is taken with the magnetometer positioned at many orientations within the field, then the measured points will map out a surface in the B system. If we assume that the axes of sensitivity of the magnetometer (with their non-zero offsets and unequal sensitivities) are mutually orthogonal, then the surface is a translated ellipsoid. The calibration function we seek transforms this ellipsoid into a unit sphere centred on the origin; applying the calibration to any given point will produce the corresponding orientation vector.

The calibration data are best collected on the glacier surface where the magnetic environment can be controlled. The operator, stripped of magnetic clothing, holds the inclinometer at a succession of orientations. At each position, the inclinometer is held steady while the three magnetometer outputs are recorded. The orientations are chosen such that the Earth's magnetic field intersects the magnetometer sensor from as many directions as the patience of the operator allows. The specific calibration orientations are not important, so a hand-held calibration can be used. In addition to instrument error, reading errors result from fluctuations in the Earth's magnetic field during the calibration and movement of the inclinometer as data are recorded (the band width of the magnetometer is $5 \mathrm{~Hz}$ and the three components are measured sequentially within a span of $114 \mathrm{~ms})$.

Typically, about 200-500 calibration triplets are collected. A calibration transformation that maps a data point $\mathbf{M}_{(\mathrm{B})}$ on this ellipsoid on to a unit sphere centred on the origin is given by

$$
\left[\begin{array}{l}
m_{x(\mathrm{~B})} \\
m_{y(\mathrm{~B})} \\
m_{z(\mathrm{~B})}
\end{array}\right]=\left[\begin{array}{ccc}
S_{x x} & 0 & 0 \\
0 & S_{y y} & 0 \\
0 & 0 & S_{z z}
\end{array}\right]\left[\begin{array}{l}
M_{x(\mathrm{~B})}+T_{x} \\
M_{y(\mathrm{~B})}+T_{y} \\
M_{z(\mathrm{~B})}+T_{z}
\end{array}\right]
$$

where $\mathbf{T}$ is an offset vector, $\mathbf{S}$ is a scaling matrix, and $\mathbf{m}_{(\mathrm{B})}$ is the transformed point. The six free parameters $\left(S_{x x}, S_{y y}, S_{z z}, T_{x}, T_{y}, T_{z}\right)$ are determined by minimizing the objective function

$$
\Phi=\sum_{i=1}^{n}\left[m_{x}^{2}(\mathrm{~B}) i+m_{y}^{2}(\mathrm{~B}) i+m_{z}^{2}(\mathrm{~B}) i-1\right]^{2}
$$

for the $n$ transformed calibration triplets $\left(S_{x x}, S_{y y}\right.$ and $S_{z z}$ are non-negative). An algebraic approach to solving the minimization is algebraically horrific, if not intractable, so we use an iterative six-dimensional simplex algorithm (Press and others, 1986, p. 289). For the UBC inclinometer, the transformed calibration data fit a unit sphere to within $2 \%$. Removing the constraint on magnetometer-axis orthogonality by allowing non-zero off-diagonal terms in calibration matrix $\mathbf{S}$ results in a negligible improvement in fit, at the expense of greatly increasing the geometrical complexity of data analysis.

\section{Transformations}

In this section, we discuss the transformations that are applied to data from the UBC inclinometer. Some of these transformations are generic in nature and can be used with all inclinometers.

In order to simplify the analysis, the scaled magnetic vector $\mathbf{m}_{(\mathrm{B})}$ must be rotated about the inclinometer axis from the B system into the T system (Fig. 2). The angle $\Omega$, measured from the $x_{\mathrm{B}}$ axis to the $x_{\mathrm{T}}$ axis, is calculated as the sum of two intermediate angles. The angle between the $x_{\mathrm{T}}$ and the $x_{\mathrm{C}}$ axes can be measured accurately on the tilt calibration frame. The angle between the $x_{\mathrm{C}}$ and $x_{\mathrm{B}}$ axes is not as well constrained. Repeated sightings on known geographical reference points are made through a telescope clamped to the machined facet on the body of the inclinometer. By reconciling the magnetic declination published on the 1:50000 topographic map (Energy, Mines and Resources, Canada map sheet $115 \mathrm{~F} / 1$, Edition 1, 1987; covers Trapridge Glacier, Yukon) with the coordinates of the reference points and the coordinates of the observation site, the value of this angle can be computed to within $\pm 0.5^{\circ}$. Weather permitting, these reference sightings are made before and after each hole is logged. This allows correction for temporal variations in magnetic declin-ation caused by geomagnetic disturbances.

The calibrated magnetic vector $\mathbf{m}_{(\mathrm{B})}$, from Equation (2), is nominally a unit vector, but errors in the data will result in a slightly erroneous vector magnitude. If the magnitude is not corrected, then the components of $\mathbf{m}_{(\mathrm{B})}$, and vectors derived from it, cannot be used as direction cosines. Therefore, the unit magnetic vector $\hat{\mathbf{m}}$ is defined by rotating and normalizing $\mathbf{m}_{(\mathrm{B})}$ using the equation

$$
\left[\begin{array}{l}
m_{x} \\
m_{y} \\
m_{z}
\end{array}\right]=\frac{1}{\left|m_{(\mathrm{B})}\right|}\left[\begin{array}{ccc}
\cos \Omega & \sin \Omega & 0 \\
-\sin \Omega & \cos \Omega & 0 \\
0 & 0 & 1
\end{array}\right]\left[\begin{array}{l}
m_{x(\mathrm{~B})} \\
m_{y(\mathrm{~B})} \\
m_{z(\mathrm{~B})}
\end{array}\right]
$$

where $\left|\mathbf{m}_{(B)}\right|$ denotes the scalar length of vector $\mathbf{m}_{(B)}$.

Examination of Figure 3 reveals that, in the case where $\hat{\mathbf{m}}$ is the full magnetic field vector, there is redundancy in the data. The three degrees of freedom representing the orientation of the inclinometer body are constrained by the four degrees of freedom embodied in the components of $\hat{\mathbf{m}}$ and $\hat{\mathbf{n}}$, which in turn are derived from the five instrument readings $\left(I_{x}, I_{y}, M_{x(\mathrm{~B})}, M_{y(\mathrm{~B})}\right.$ and $\left.M_{z(\mathrm{~B})}\right)$. The two additional degrees of freedom governed by the instrument readings are the magnitude of the magnetic field vector (lost in normalizing $\hat{\mathbf{m}}$ ) and the spin orientation of the inclinometer on its axis (in our analysis, this information is discarded). For the UBC inclinometer, the value of $\hat{\mathbf{n}}$ is far better determined than $\hat{\mathbf{m}}$, so we use $\hat{\mathbf{n}}$ to define the inclination angle of the $z_{\mathrm{T}}$ axis. Vector $\hat{\mathbf{m}}$ is used to constrain the azimuth of the $z_{\mathrm{T}}$ axis about vector $\hat{\mathbf{n}}$. The functions of $\hat{\mathbf{m}}$ and $\hat{\mathbf{n}}$ can be exchanged but, were $\hat{\mathbf{m}}$ used as the inclination reference, accuracy would be lost. Note that data from magnetically oriented inclinometers become indeterminate when $\hat{\mathbf{m}}$ and $\hat{\mathbf{n}}$ are parallel or anti-parallel.

Our objective now is to find a set of Eulerian transformation angles $\phi, \theta$ and $\psi$ (see Appendix A) which will map $\hat{\mathbf{m}}$, as expressed in the $\mathrm{T}$ system, on to the $\mathrm{U}$ system such that the horizontal projection of $\hat{\mathbf{m}}$ has the proper magnetic declination $d$. By casting the transformation in this way, we avoid directly implicating the $\mathrm{H}$ system. The transformation must also map $\hat{\mathbf{n}}$, as expressed in the $\mathrm{T}$ system, on to the vertical in the $\mathrm{U}$ system. We will treat the $U$ system as unprimed and the $T$ system as primed (Appendix A, Equation (A2)). 
The angle $\theta$ defines the net tilt of the $z_{\mathrm{T}}$ axis with respect to the vertical $z_{\mathrm{U}}$ axis. This net tilt is simply

$$
\theta=\cos ^{-1} n_{z} \text {. }
$$

The angle $\psi$ controls the relative contribution of the two tilt angles to the net tilt, defined as

$$
\psi=\tan ^{-1}\left(\frac{n_{x}}{n_{y}}\right)
$$

The angle $\phi$ rotates the T system about the $z_{\mathrm{U}}$ axis; in other words, $\phi$ is the Eulerian angle that controls the declination of the transformed magnetic vector. Given $\theta$ and $\psi$, the value of $\phi$ must satisfy

$$
\left[\begin{array}{c}
F \sin d \\
F \cos d \\
\left(1-F^{2}\right)^{\frac{1}{2}}
\end{array}\right]=\mathbf{A}^{T}\left[\begin{array}{l}
m_{x} \\
m_{y} \\
m_{z}
\end{array}\right]
$$

where $0<F \leq 1$ is the magnitude of $\hat{\mathbf{m}}$ when projected on to the horizontal plane and $d$ is the magnetic declination east of UTM north. This system of equations has an explicit solution for $\phi$ and $F$, but the algebra can be simplified considerably by first solving for the case where $d=0$ and subsequently adjusting the solution. This trick results in an indirect association with the $\mathrm{H}$ system. The equation to solve becomes

$$
\left[\begin{array}{c}
0 \\
F \\
\left(1-F^{2}\right)^{\frac{1}{2}}
\end{array}\right]=\mathbf{A}^{T}\left[\begin{array}{l}
m_{x} \\
m_{y} \\
m_{z}
\end{array}\right]
$$

and the solution is

$$
\phi=\tan ^{-1}\left[\frac{m_{x} \cos \psi-m_{y} \sin \psi}{\left(m_{x} \sin \psi+m_{y} \cos \psi\right) \cos \theta-m_{z} \sin \theta}\right]
$$

$$
\begin{aligned}
F=[ & \left(m_{x} \cos \psi-m_{y} \sin \psi\right)^{2} \\
& +\cos ^{2} \theta\left(m_{y} \cos \psi-m_{x} \sin \psi\right)^{2} \\
& \left.+m_{z} \sin ^{2} \theta\left(m_{y} \cos \psi-m_{x} \sin \psi\right)+m_{z}^{2} \sin ^{2} \theta\right]^{1 / 2} .
\end{aligned}
$$

The non-zero declination $d$ is reinstated by modifying Equation (9) to give

$$
\phi=\tan ^{-1}\left[\frac{m_{x} \cos \psi-m_{y} \sin \psi}{\left(m_{x} \sin \psi+m_{y} \cos \psi\right) \cos \theta-m_{z} \sin \theta}\right]-d .
$$

Equations (9) and (11) are curiously insensitive to the value of $m_{z}$ in the sense that a value of $\hat{\mathbf{m}}$ derived from a gimbal-mounted compass (i.e. $\hat{\mathbf{m}}$ is horizontal) will give the same value for $\phi$ as a value of $\hat{\mathbf{m}}$ representing the full Earth field, but this insensitivity is expected. Both gimbalmounted and full-field magnetometers are equally adept at determining magnetic bearing.

As the final step in the data-transformation procedure, the orientation vector $\hat{\mathbf{t}}$ of the inclinometer (which corresponds to the $z_{\mathrm{T}}$ axis), as expressed in the $\mathrm{U}$ system, is computed by the transformation

$$
\left[\begin{array}{l}
t_{x} \\
t_{y} \\
t_{z}
\end{array}\right]=\mathbf{A}^{T}\left[\begin{array}{l}
0 \\
0 \\
1
\end{array}\right]=\left[\begin{array}{c}
\sin \theta \sin \phi \\
-\sin \theta \cos \phi \\
\cos \theta
\end{array}\right] .
$$

The Eulerian transformation matrix $\mathbf{A}$ is defined in Table 1 and by Equations (5), (6) and (11). For this analysis the value of $F$ is irrelevant.

\section{Universal application of transformations}

Note that Equations (5), (6), (9) and (12) apply to any inclinometry data that can be expressed in terms of a tilt vector $\hat{\mathbf{n}}$ and an orientation vector $\hat{\mathbf{m}}$. This includes data from tracked and gyroscopically oriented inclinometers.

\section{Inverse problem}

In this section, we develop a general theory, applicable to all inclinometers, for computing a continuous borehole trajectory based on discrete measurements of borehole depth and inclinometer orientation. Before considering the details of this step, it is appropriate to consider the relationship between the inclinometer orientation and the borehole axis.

The simplest assumed relationship is that the inclinometer is always parallel to the borehole axis. Although this presumption is erroneous, without additional data describing the borehole geometry and centring device geometry, it is difficult to improve upon. At any given station along the borehole, the inclinometer axis lies along a straight line drawn between the centring devices. If the two centring devices operate identically, the borehole has smooth walls and constant diameter, and the curvature of the borehole is a circular arc, then the inclinometer axis will lie parallel to the borehole axis at a point midway between the centring devices. In practice, these three conditions are rarely satisfied. It is here that tracked inclinometers exhibit improved performance over instruments such as the UBC tool; the groove-tracking wheels can accurately position the inclinometer in the centre of the casing and the casing provides both smooth walls and regular borehole geometry. The trajectory computation method described below will assume that the borehole and inclinometer axes coincide; the

Table 1. The Eulerian transformation matrix

$$
\mathbf{A}=\left(\begin{array}{ccc}
\cos \psi \cos \phi-\cos \theta \sin \phi \sin \psi & \cos \psi \sin \phi+\cos \theta \cos \phi \sin \psi & \sin \psi \sin \theta \\
-\sin \psi \cos \phi-\cos \theta \sin \phi \cos \psi & -\sin \psi \sin \phi+\cos \theta \cos \phi \cos \psi & \cos \psi \sin \theta \\
\sin \theta \sin \phi & -\sin \theta \cos \phi & \cos \theta
\end{array}\right)
$$


sensitivity of the method to positioning error will be discussed at the end of the paper.

The task of computing a continuous borehole trajectory from a set of discrete tilt and azimuth measurements constitutes an inverse problem. An infinite number of possible trajectory solutions exist that will satisfy a given data set exactly. An infinite number of additional solutions exists that do not satisfy the data exactly, but which do fall within the error bounds of the data. A single solution of the first type can be isolated by placing appropriate geometrical constraints on the trajectory; the solution to the inverse problem becomes an interpolation scheme. All treatises of which we are aware, this paper included, use geometrical constraints to choose an exact solution that will hopefully reflect the true trajectory. This method for solving an inverse problem differs from traditional methods in that an a priori structural model is solely responsible for isolating one of the possible solutions. Traditional methods can find solutions of the second type by setting an objective function, such as minimizing the rate of change of borehole tilt, and by allowing for assessment of error in the data. Data smoothing is another possible approach to finding inexact trajectory solutions.

Many methods for interpolating slope angles have been discussed in the petroleum industry literature. The

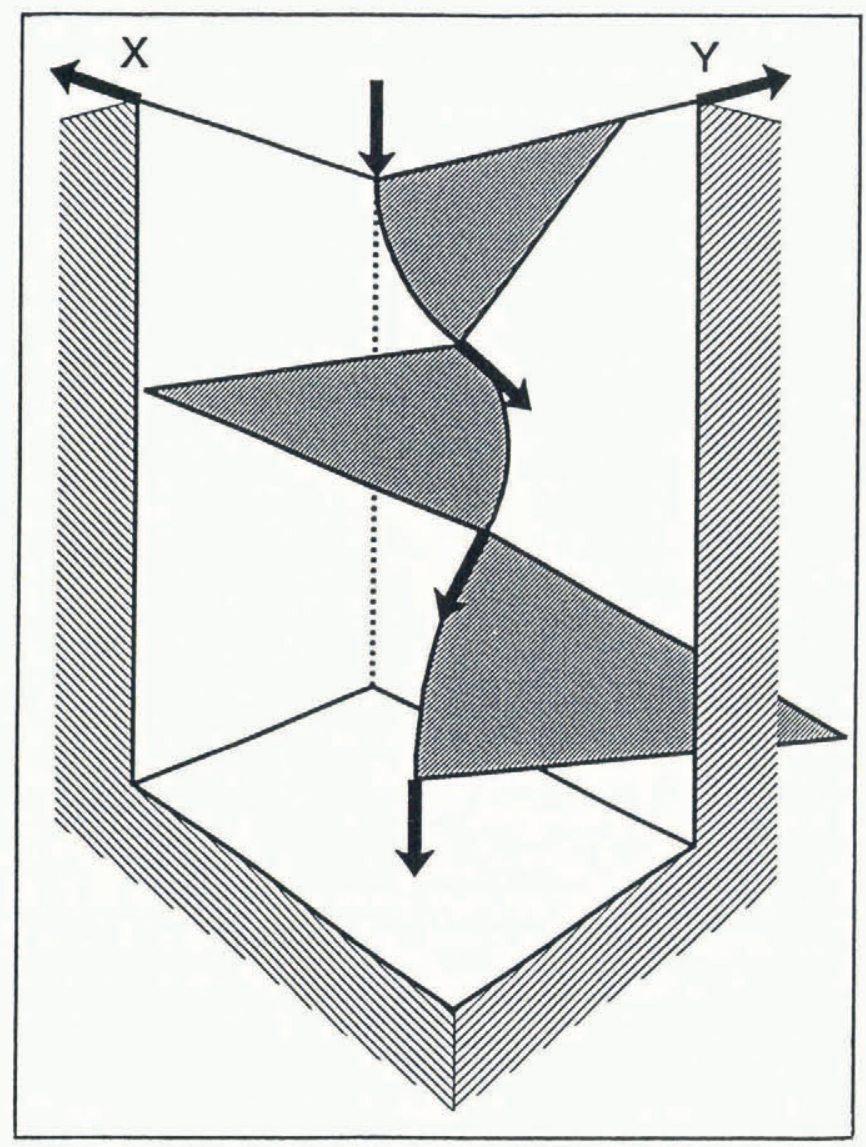

Fig. 4. A perspective view of the circular-arc interpolation model. Between pairs of measurement stations, the borehole is assumed to follow a circular trajectory. The arc length is constrained by the measured distance between stations, and the plane of the arc is defined by the instrument-orientation vectors at the end points of the arc. These orientation vectors are tangential to the arc. "terminal-angle tangential method" is often mentioned as the best-known interpolation method: over any station interval, the orientation of the inclinometer axis is assumed to be equal to that of the lower station. In effect, no interpolation is performed and the slope of the computed trajectory is discontinuous at every station. Because of its faulty representation of inclinometer motion, this method results in appreciable error. Walstrom and others (1972) discussed five different methods for interpolating the tilt angle and azimuth between stations. The underlying premise of many of their mathematical models is that tilt angle and azimuth can be treated as independent quantities. This premise does not always hold true, as is revealed by considering the radius-of-curvature method developed by Wilson (1968) and later expanded by Rivero (1971). Their method maps the borehole trajectory on to the surface of a vertical cylinder such that the desired azimuth and tilt are preserved at the end points of the spiral segment. The resulting trajectory is not independent of the coordinate system in which the projection is made, indicating that in this case the tilt angle and azimuth cannot be treated separately. Angle-interpolation methods are also cluttered with special treatment for the multivalued nature of the inverse trigonometric functions.

We assume that the path of the inclinometer between stations can be described by a series of circular arc sections (Fig. 4). Figure 5 shows one of these arcs in detail. The end points of the $\operatorname{arc}\left(\mathbf{P}_{1}, \mathbf{P}_{2}\right)$ are tangential to the normalized orientation vectors $\left(\hat{\mathbf{t}}_{1}, \hat{\mathbf{t}}_{2}\right)$, and the length of the $\operatorname{arc} L$ equals the measured depth increment on the cable. Successive arcs join in a smooth manner with no discontinuities in slope, although discontinuities in the direction of slope change are present. If the position of $\mathbf{P}_{1}$ is known, then the position of $\mathbf{P}_{2}$ or any intermediate point $\mathbf{P}_{3}$ along the arc can be computed. This method has been discussed by Zaremba (1973), but

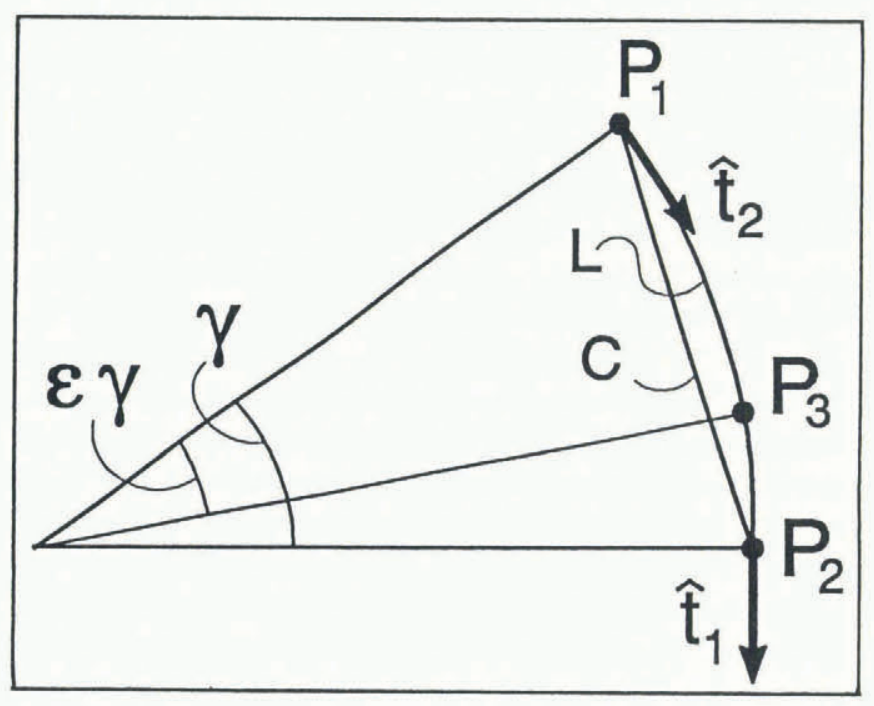

Fig. 5. A detailed view of one circular arc in the borehole trajectory. Knowing the position of the starting point $\mathbf{P}_{1}$, the tangent vectors $\hat{\mathbf{t}}_{1}$ and $\hat{\mathbf{t}}_{2}$, and the length of the arc $L$, the position of the end point $\mathbf{P}_{2}$ can be computed. The position of any intermediate point $\mathbf{P}_{3}$ on the arc can also be computed. The angle $\gamma$ is defined by $\hat{\mathbf{t}}_{1}$ and $\hat{\mathbf{t}}_{2}$. 
Zaremba derived an unnecessarily complicated solution. Our solution for $\mathbf{P}_{2}$ is

$$
\mathbf{P}_{2}=\mathbf{P}_{1}+\frac{L}{\gamma} \tan \left(\frac{\gamma}{2}\right)\left[\hat{\mathbf{t}}_{1}+\hat{\mathbf{t}}_{2}\right]
$$

where

$$
\cos \gamma=\hat{\mathbf{t}}_{1} \cdot \hat{\mathbf{t}}_{2} .
$$

In the limit, where $\hat{\mathbf{t}}_{2} \rightarrow \hat{\mathbf{t}}_{1}$ (the anti-parallel case is unlikely), the circular arc will have infinite radius $(\gamma \rightarrow 0)$, and the solution for $\mathbf{P}_{2}$ becomes

$$
\mathbf{P}_{2}=\mathbf{P}_{1}+L \hat{\mathbf{t}}_{1} \text {. }
$$

A full derivation of the solution is found in Appendix B. A solution for a point $\mathbf{P}_{3}$ at an arbitrary position along the arc is also given. The trajectory computed by this circular-arc method is independent of the coordinate system orientation. The trajectory of the borehole is reconstructed by successively applying Equations (13) and (15), as appropriate, beginning at the glacier surface and working downward.

\section{SENSITIVITY}

The trajectory of the borehole as determined by the circular-arc method, or by any other interpolation method, is inherently incorrect - even if the boreholeorientation data are error-free. This is because the continuous borehole trajectory is sampled at a finite number of points. In the case of the UBC inclinometer, we do not believe that sampling density is a major source of error because our $1 \mathrm{~m}$ sampling interval is short compared to the length of the inclinometer and the drill stem used to drill the holes; we do not expect perturbations in the borehole trajectory on a scale smaller than $1 \mathrm{~m}$. For this dense sampling interval, and given error-free borehole-orientation data, the divergence between the true trajectory and the computed trajectory is expected to be at most a few centimetres. Problems arise because the borehole-orientation data are not error-free.

The UBC inclinometer has logged 125 boreholes during the 1989 and 1990 field seasons at Trapridge Glacier, Yukon. Each hole was logged at least twice. By comparing the inclinometry results, we estimate that the instrument locates the bottom of a $70 \mathrm{~m}$ hole to within 20 $30 \mathrm{~cm}$. The error tends to be largest in the azimuthal sense; the radial deviation of a borehole is determined to within $15 \mathrm{~cm}$. Based on this evidence, we identify two major sources of error: random-positioning error of the inclinometer in the borehole and systematic orientation error. Superimposed on these error terms are the measurement errors of the tilt sensors and magnetometer.

Accurate positioning of a non-tracked inclinometer within a borehole is dependent on the texture of the borehole wall and the performance of the centring devices. Glacier borehole walls are not necessarily smooth and the centring devices allow the inclinometer to cant relative to the borehole axis. The discrepancy between the axis of the borehole and the axis of the inclinometer is estimated at $\pm 0.5^{\circ}$. This estimate is based on the diameter of the borehole and that of the inclinometer with the centring-devices fully compressed. It is also likely that the inclinometer tends to underestimate the borehole tilt since tension in the cable and the instrument weight work to force the instrument towards a vertical orientation. As this effect depends on the lay of the cable and the local hole geometry, it is impractical to quantify; we satisfy ourselves with the $\pm 0.5^{\circ}$ positioning error. The tilt-sensor error (less than one part in 10000) is neglected. The magnetometer error is appreciable and amounts to $\pm 2 \%$ on the $x_{\mathrm{B}}$ and $y_{\mathrm{B}}$ axes, and $\pm 3 \%$ on the $z_{\mathrm{B}}$ axis. These estimates are based on the calibration fit and the specifications for the magnetorneter. Neglecting stretch in the cable, the error in positioning along the borehole is estimated at $1 \mathrm{~cm}$ for each $1 \mathrm{~m}$ interval.

The effects of the random errors can be modelled using Monte Carlo techniques. Synthetic data are generated for a sequence of stations with error terms superimposed on the "correct" data. The standard deviation of the Gaussian-distributed error for each reading is given above. Relative contributions to the net deviation can be examined by selectively removing the error terms. Figure 6 shows the results from a series of Monte Carlo tests on a 70 station hole ( $69 \mathrm{~m}$ long). The figure shows horizontal maps of the computed bottom locations for each Monte Carlo test. The underlying model is a linear hole dipping $10^{\circ}$ to the south with the top at the origin. Each test contains 1000 runs through the hole. Figure $6 \mathrm{a}$ shows the effects of applying all error terms simultaneously. The cross hair indicates the location of the errorfree hole bottom at $11.98 \mathrm{~m}$ south. Note the slight stretching of the "data cloud" in the azimuthal (eastwest) sense and the offset towards the north (origin). Both of these effects are primarily due to error introduced by the magnetometer, as can be demonstrated by removing the magnetometer and depth error (Fig. 6b). This results in a more symmetric cloud, with less noticeable offset. Figure $6 \mathrm{c}$ shows how the magnetometer introduces these two distortions. The east-west lineation is actually a short section of a circle with its centre at the origin. This is easily seen in Figure $6 \mathrm{~d}$ where gross errors $( \pm 50 \%)$ in the magnetometer over one station interval result in a circular scattering envelope. Again, the cross hair indicates the error-free solution. The foreshortening (drawing in towards the origin) observed in Figures $6 a, c$ and $d$ results from the fact that error in azimuth will always cause the radius of the interpolating circular arc to decrease; this results in a horizontal projection that is always smaller than, or equal to, the true projection. On average, error in tilt measurements also produces foreshortening (Fig. 6b). Note that, for holes of this depth, the foreshortening amounts to no more than a few centimetres. In Figure 6a, the net depth error amounts to no more than $\pm 2 \mathrm{~cm}$.

Systematic error is caused by the non-random orientation of the inclinometer as it moves through the borehole. The orientation of the inclinometer does not change greatly as it moves from station to station but, in separate passes through the hole, the general orientation of the inclinometer may be quite different. It follows that any offset error in determining the angle $\Omega$ between the $x_{\mathrm{T}}$ and $x_{\mathrm{B}}$ axes will accumulate differently in different inclinometer passes, and this will result in additional 

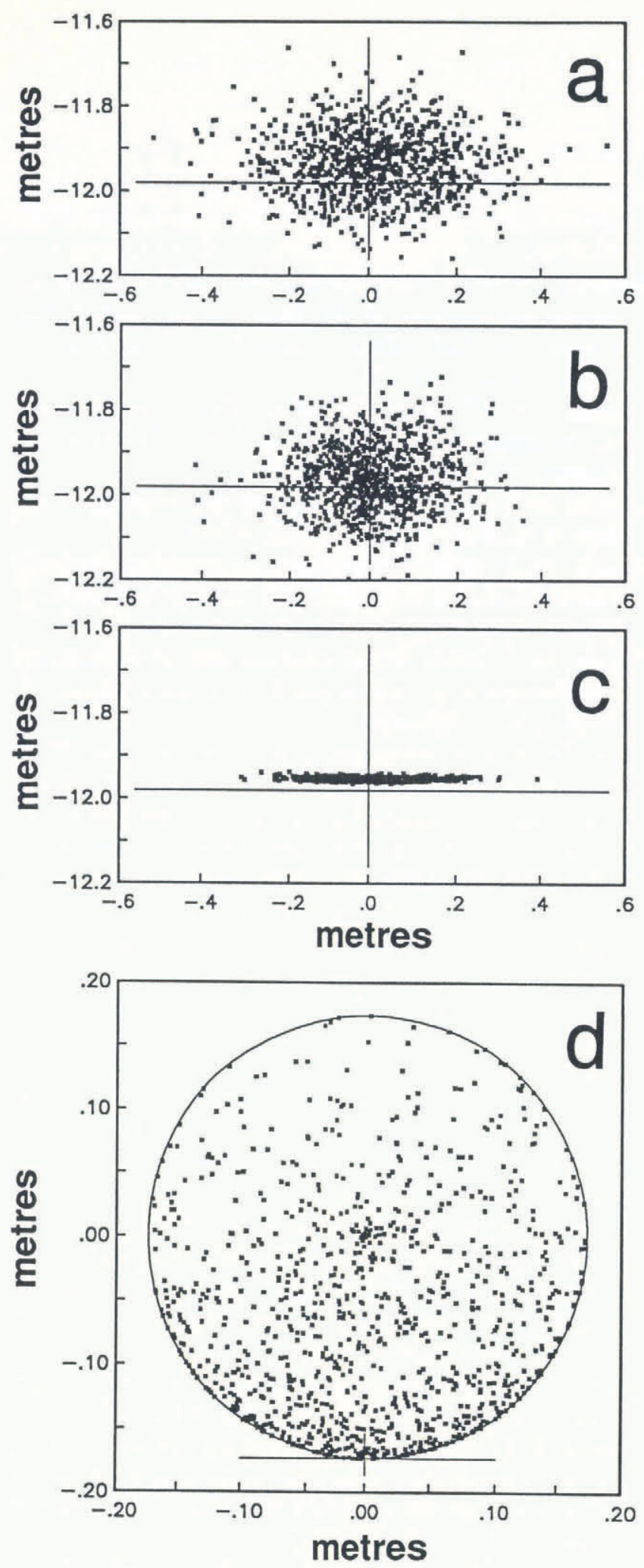

Fig. 6. Monte Carlo modelling of the sensitivity of the inclinometer toerror in its sensors is shown as a series of scatter plots. Panels (a),(b) and (c) show the horizontal deviation of the computed hole bottom from its true position for 1000 Monte Carlo runs down a $69 \mathrm{~m}$ hole dipping southward at $10^{\circ}$ (station interval of $1 \mathrm{~m}$ ). The hole top is at the origin and the true bottom position is indicated by the cross hair.(a) The combined effect of $a \pm 0.5^{\circ}$ error in tilt, a $2 \%$ error in $m_{x}$ and $m_{y}, a 3 \%$ error in $m_{z}$ and a $1 \mathrm{~cm} \mathrm{~m}^{-1}$ error in depth control. (b) The effect of tilt error alone. (c) The magnetometer error alone. (d) Scatter produced by allowing gross (50\%) error in the components of $\hat{\mathbf{m}}$ over one station interval. azimuthal error. By adjusting the value of $\Omega$ such that the azimuthal error is minimized for all holes, we can remove much of the systematic error.

Our estimated positioning error of $20-30 \mathrm{~cm}$, with slightly better radial control, is consistent with the results of the Monte Carlo test in Figure 6a. This leads us to conclude that we have a good understanding of the factors influencing the performance of our inclinometer.

\section{DISCUSSION}

Our experience with the UBC inclinometer has shown that it is an efficient and accurate instrument for glaciological work. Surveys are performed quickly and do not require any special attention to the instrument or preparation of the borehole. For these reasons, we expect that magnetically oriented inclinometers using fluxgate magnetometers will gain popularity with glaciologists. For rigorous applications, some researchers may wish to combine the better tilt accuracy of a tracked inclinometer with the additional check on orientation provided by a magnetometer.

The principal shortcoming of these instruments is that they become confused at very high magnetic latitudes. We estimate that the UBC inclinometer will not operate reliably where the dip of the magnetic field exceeds $85^{\circ}$. This constraint probably excludes its use throughout most of the Canadian Arctic archipelago and the Wilkes Coast and George V Land in Antarctica. We know that the accuracy of the magnetometer is being degraded by its close proximity to electrical currents inside the pressure tube. Redesigning the internal configuration of the instrument so as to avoid this magnetic noise would reduce the error observed in Figure 6c, and might allow the instrument to be used closer to the Earth's magnetic poles.

These restrictions aside, the error in determining the position of subglacial and englacial sensors with the UBC inclinometer is small enough for all but the most exacting experiments. We are seeking to improve the performance of the instrument, primarily by redesigning the centralizing springs. If this results in reduced error in the tilt readings, then the instrument might approach the sensitivity represented by Figure $6 \mathrm{c}$.

The circular-arc interpolation scheme is simple to apply and easy to visualize. On a $12 \mathrm{MHz}$ IBM-PCcompatible computer equipped with a numerical coprocessor, the time required to calibrate, process and plot the data from a $70 \mathrm{~m}$ borehole takes a fraction of a second. The foreshortening effect of the circular-arc method is the only drawback of which we are aware, but this effect is not unique to this interpolation method.

We expect that improvements in the accuracy of inclinometry-data inversion can be obtained in at least three ways: (1) Incorporate additional borehole geometry and centring-device geometry. (2) Study the effects of centring-device spacing relative to station spacing. (3) Introduce full inversion techniques that produce trajectories having an imperfect fit, within error, to the data. Data smoothing is one strategy for finding these imperfectly fitting trajectories, but it is important that the smoothing process does not result in violation of the 
error bounds on the data. We invite other interested parties to consider these approaches.

\section{ACKNOWLEDGEMENTS}

The authors wish to thank A.G. Fountain (USGS Denver) and P.L. Taylor (Hydro-Tech, Seattle) for lending us their inclinometer for the 1988 field season. We wish to acknowledge the contributions of B. B. Narod of Narod Geophysics for designing and building the prototype magnetometer, and $\mathrm{L}$. Theriault and $\mathrm{S}$. Cornwallace of Slope Indicator Canada Limited for assembling the combined magnetometer/accelerometer instrument. Funding for the construction of the inclinometer was provided by an equipment grant from the Natural Sciences and Engineering Research Council of Canada (NSERC). Field work was supported by the Canadian Northern Studies Trust of the Association of Canadian Universities for Northern Studies, NSERC and the Northern Science Training Program of the Department of Indian Affairs and Northern Development.

\section{REFERENCES}

Brand, G., V. Pohjola and R. LeB. Hooke. 1987. Evidence for a till layer beneath Storglaciären, Sweden, based on electrical resistivity measurements. F. Glaciol., 33(115), 311-314.

Garfield, D.E. and H. T. Ueda. 1976. Resurvey of the "Byrd" station, Antarctica, drill hole. 7. Glaciol., 17(75), 29-34.

Gerrard, J.A.F., M.F. Perutz and A. Roch. 1952. Measurement of the velocity distribution along a vertical line through a glacier. Proc. R. Soc., Ser. A, 213(1115), 546-558.

Hooke, R. LeB. 1973. Structure and flow in the margin of the Barnes Ice Cap, Baffin Island, N.W.T., Canada. J. Glaciol., 12(66), 423-438.

Hooke, R. LeB. and B. Hanson. 1986. Borehole deformation experiments, Barnes Ice Cap, Canada. Cold Reg. Sci. Technol., 12(3), 261-276.

Hooke, R. LeB., P. Holmlund and N. R. Iverson. 1987. Extrusion flow demonstrated by bore-hole deformation measurements over a riegel, Storglaciären, Sweden. $\mathcal{J}$. Glaciol., 33(113), 72-78.

Levanto, A.E. 1959. A three-component magnetometer for small drill-holes and its use in ore prospecting. Geophys. Prospect., 7(2), 183-195.

Miller, M. M. 1958. Phenomena associated with the deformation of a glacier bore-hole. International Association of Scientific Hydrology Publication 46, (General Assembly of Toronto 1957 - Snow and Ice), 437-452.

Narod, B. B. and R.D. Russell. 1984. Steady-state characteristics of the capacitively loaded flux gate sensor. IEEE Trans. Magn., MAG-20(4), 592-597.

Narod, B. B., J. R. Bennest, J. O. Strom-Olsen, F. Nezil and R.A. Dunlap. 1985. An evaluation of the noise performance of $\mathrm{Fe}, \mathrm{Co}, \mathrm{Si}$, and $\mathrm{B}$ amorphous alloys in ring-core fluxgate magnetometers. Can. J. Phys., 63(11), 1468-1472.

Nye, J. F. 1952. The mechanics of glacier flow. F. Glaciol.,
2(12), 82-93.

Nye, J.F. 1953. The flow law of ice from measurements in glacier tunnels, laboratory experiments and the Jungfraufirn borehole experiment. Proc. $R$. Soc., Ser. A, 219(1139), 477-489.

Nye, J.F. 1957. The distribution of stress and velocity in glaciers and ice-sheets. Proc. R. Soc., Ser. A, 239(1216), 113-133.

Paterson, W. S. B. 1983. Deformation within polar ice sheets: an analysis of the Byrd Station and Camp Century borehole-tilting measurements. Cold Reg. Sci. Technol., 8(2), 165-179.

Paterson, W. S. B. and J. C. Savage. 1963. Measurements on Athabasca Glacier relating to the flow law of ice. $\mathcal{J}$. Geophys. Res., 68(15), 4537-4543.

Perutz, M.F. 1947. Report on problems relating to the flow of glaciers. 7 . Glaciol., 1 (2), 47-51.

Perutz, M.F. 1949. Direct measurement of the velocity distribution in a vertical profile through a glacier. $\mathcal{f}$. Glaciol., 1(5), 249.

Perutz, M.F. 1950. Direct measurement of the velocity distribution in a vertical profile through a glacier. $\mathcal{F}$. Glaciol., 1(7), 382-383.

Press, W. H., B. P. Flannery, S. A. Teukolsky and W. T. Vetterling. 1986. Numerical recipes: the art of scientific computing. Cambridge, Cambridge University Press.

Raymond, C. F. 1971 a. A new bore-hole inclinometer. J. Glaciol., 10(58), 127-132.

Raymond, C.F. 1971b. Determination of the threedimensional velocity field in a glacier. F. Glaciol., 10(58), 39-53.

Raymond, C.F. 1971c. Flow in a transverse section of Athabasca Glacier, Alberta, Canada. 7. Glaciol., 10(58), 55-84.

Rivero, R. T. 1971. Use of the curvature method to determine true vertical reservoir thickness. J. Pet. Technol., 23, 491-496.

Russell, R. D., B. B. Narod and F. Kollar. 1983. Characteristics of the capacitively loaded flux gate sensor. IEEE Trans. Magn., MAG-19(2), 126-130.

Savage, J. C. and W.S. B. Paterson. 1963. Borehole measurements in the Athabasca Glacier. 7. Geophys. Res., 68(15), 4521-4536.

Sharp, R. P. 1953. Deformation of bore hole in Malaspina Glacier, Alaska. Bull. Geol. Soc. Am., 64, 97-100.

Shreve, R. L. 1961. The borehole experiment on Blue Glacier, Washington. International Association of Scientific Hydrology Publication 54, (General Assembly of Helsinki 1960 - Snow and Ice), 530-531.

Shreve, R. L. and R. P. Sharp. 1970. Internal deformation and thermal anomalies in lower Blue Glacier, Mount Olympus, Washington, U.S.A. J. Glaciol., 9(55), 65-86.

Walker, B. H. 1986. Factors controlling hole angle and direction. J. Pet. Technol., 38(12), 1171-1173.

Walstrom, J. E., R. P. Harvey and H. D. Eddy. 1972. A comparison of various directional survey models and an approach to model error analysis. 7. Pet. Technol., 24, 935-943.

Wilson, G.J. 1968. An improved method for computing directional surveys. 7. Pet. Technol., 20, 871-876.

Wyckoff, R. D. 1948. The Gulf airborne magnetometer. Geophysics, 13(2), 182-208. 
Zaremba, W. A. 1973. Directional survey by the circular arc method. F. Soc. Pet. Eng., 13(1), 5-11.

The accuracy of references in the text and in this list is the responsibility of the authors, to whom queries should be addressed.

\section{APPENDIX A}

\section{EULERIAN ANGLES}

The relationship between two arbitrarily rotated coordinate systems that share the same origin can be uniquely described using Eulerian angles. Figure 7 shows how a triplet of Eulerian angles $(\phi, \theta, \psi)$ defines the transformation between any two righthanded Cartesian coordinate systems. The transformation is achieved by three successive righthanded rotations about specified axes. Defined as a matrix operation, the transformation from the unprimed coordinate system to the primed is

$$
x^{\prime}=\mathbf{A} x
$$

where $x$ and $x^{\prime}$ are coordinates of a point in $\Re^{3}$ and $\mathbf{A}$ is defined in Table 1. Because $\mathbf{A}$ is an orthogonal matrix, $\mathbf{A}^{-1}=\mathbf{A}^{\mathrm{T}}$, so the inverse transformation $x=\mathbf{A}^{-1} x^{\prime}$ is simply

$$
x=\mathbf{A}^{\mathrm{T}} x^{\prime} .
$$

The inverse matrix $\mathbf{A}^{-1}$ satisfies $\mathbf{A A}^{-1}=\mathbf{I}$, where $\mathbf{I}$ is the identity matrix, and the transposed matrix $\mathbf{A}^{\mathrm{T}}$ is obtained by writing the rows of $\mathbf{A}$, in order, as columns.

This level of complexity is only necessary when moving from an instrument-based coordinate system to a map-coordinate system. Transformations between coordinate systems within these two groups is accomplished by simple rotation about the common $z$ axis $(\theta=\phi=0)$.

\section{APPENDIX B}

\section{INTERPOLATION SGHEME}

We derive solutions for $\mathbf{P}_{2}$ and $\mathbf{P}_{3}$ given the initial position $\mathbf{P}_{1}$ and the unit tangent vectors to the circular arc $\hat{\mathbf{t}}_{1}$ and $\hat{\mathbf{t}}_{2}$ (Fig. 5). Consider the general problem of solving for the intermediate co-planar vector between two non-parallel unit vectors (Fig. 8). The partitioning factor $\epsilon$ ranges between 0 and 1 . The unit vector $\hat{\mathbf{t}}_{3}$ is defined by the linear combination

$$
\hat{\mathbf{t}}_{3}=k_{1} \hat{\mathbf{t}}_{1}+k_{2} \hat{\mathbf{t}}_{2}
$$

where $k_{1}$ and $k_{2}$ are appropriate scalar values. The solutions for $k_{1}$ and $k_{2}$ must satisfy

$$
\begin{gathered}
\hat{\mathbf{t}}_{1} \cdot \hat{\mathbf{t}}_{3}=\cos (\epsilon \gamma) \\
\hat{\mathbf{t}}_{2} \cdot \hat{\mathbf{t}}_{3}=\cos [(1-\epsilon) \gamma]
\end{gathered}
$$

where

$$
\cos \gamma=\hat{\mathbf{t}}_{1} \cdot \hat{\mathbf{t}}_{2} .
$$

Equations (B2) and (B3) ensure that $\hat{\mathbf{t}}_{3}$ is a unit vector co- planar with $\hat{\mathbf{t}}_{1}$ and $\hat{\mathbf{t}}_{2}$. The solutions are

$$
\begin{gathered}
k_{1}=\frac{\cos (\epsilon \gamma)-\cos \gamma \cos [(1-\epsilon) \gamma]}{\sin ^{2} \gamma} \\
k_{2}=\frac{\sin (\epsilon \gamma)}{\sin \gamma} .
\end{gathered}
$$

In the special case of bisection $(\epsilon=0.5)$,

$$
k_{1}=k_{2}=\frac{1}{2 \cos (\gamma / 2)} \text {. }
$$

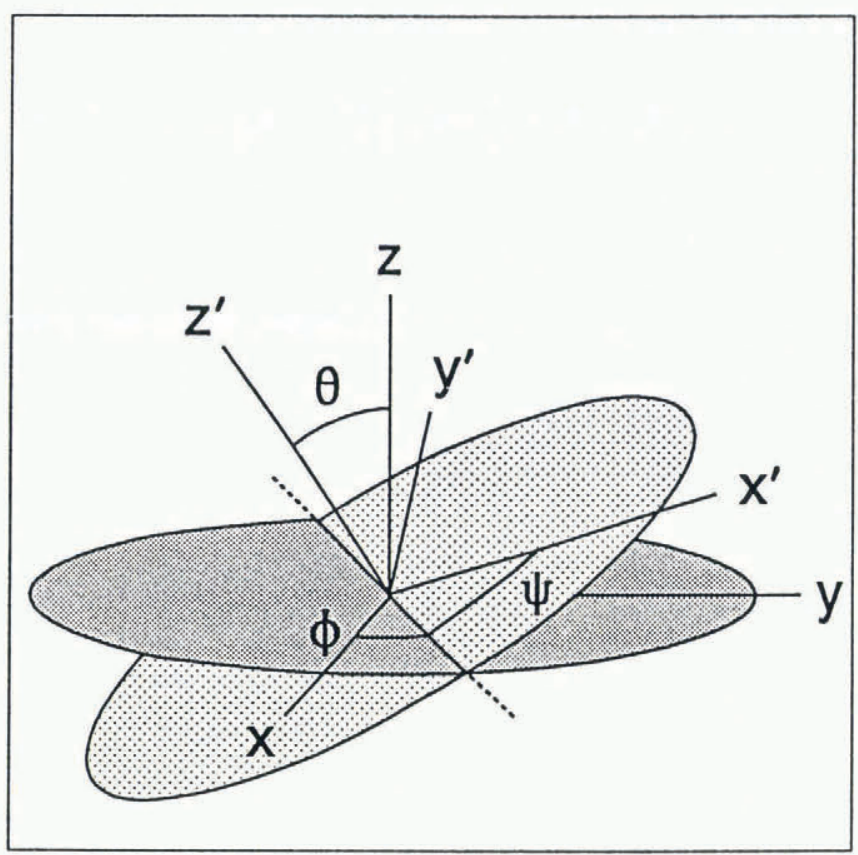

Fig. 7. A set of Eulerian angles describes the transformation between two righthanded coordinate systems sharing a common origin. The transformation from an unprimed to a primed coordinate system is defined as a set of three righthanded rotations about specified axes. The three rotations are: (1) $A$ rotation by angle $\phi$ about the $z$ axis. (2) $A$ rotation by angle $\theta$ about the intermediate $x$ axis (dotted line). (3) $A$ rotation by angle $\psi$ about the $z$ axis.

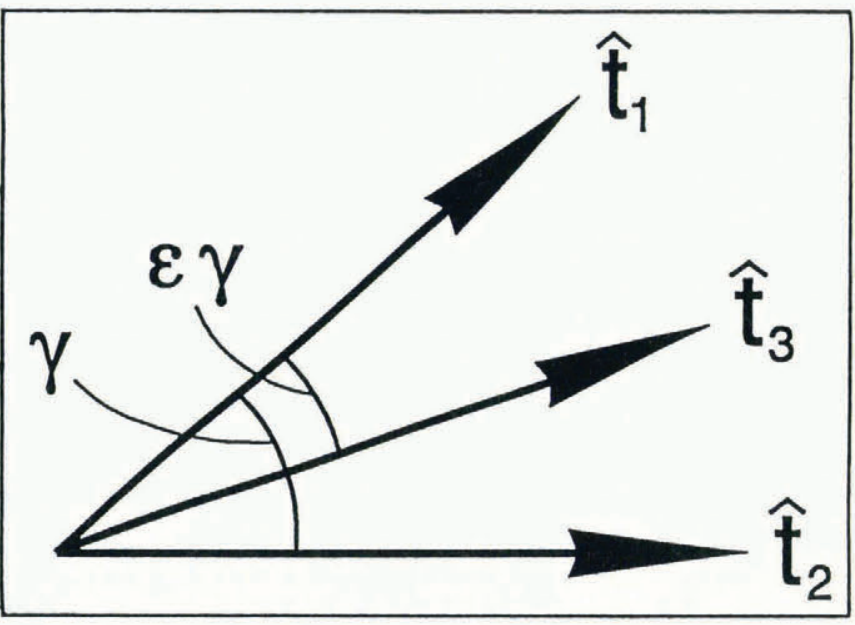

Fig. 8. The intermediate co-planar unit vector $\hat{\mathbf{t}}_{3}$ between two arbitrary non-parallel unit vectors $\hat{\mathbf{t}}_{1}$ and $\hat{\mathbf{t}}_{2}$ is shown. The value of $0 \leq \epsilon \leq 1$ defines the position of the intermediate vector. 
In Figure 5, the chord drawn between $\mathbf{P}_{1}$ and $\mathbf{P}_{2}$ bisects the two vectors $\hat{\mathbf{t}}_{1}$ and $\hat{\mathbf{t}}_{2}$. The length of the chord is given by

$$
C=2\left(\frac{L}{\gamma}\right) \sin \left(\frac{\gamma}{2}\right)
$$

and Equations (B1) and (B7) yield the unit vector along the chord

$$
\hat{\mathbf{c}}=\frac{\hat{\mathbf{t}}_{1}+\hat{\mathbf{t}}_{2}}{2 \cos (\gamma / 2)}
$$

so that we can write the solution for $\mathbf{P}_{2}$ as

$$
\mathbf{P}_{2}=\mathbf{P}_{1}+\frac{L}{\gamma} \tan \left(\frac{\gamma}{2}\right)\left[\hat{\mathbf{t}}_{1}+\hat{\mathbf{t}}_{2}\right] .
$$

The position at any intermediate position $\mathbf{P}_{3}$, located at some fractional distance $0 \leq \epsilon \leq 1$ along the $\operatorname{arc}$ from $\mathbf{P}_{1}$ is given by a similar construction as

$$
\mathbf{P}_{3}=\mathbf{P}_{1}+\frac{L}{\gamma} \tan \left(\frac{\epsilon \gamma}{2}\right)\left[\left(k_{1}+1\right) \hat{\mathbf{t}}_{1}+k_{2} \hat{\mathbf{t}}_{2}\right]
$$

where $\gamma, k_{1}$ and $k_{2}$ are defined by Equations (B4), (B5) and (B6).

In the special case where $\hat{\mathbf{t}}_{1}=\hat{\mathbf{t}}_{2}$ (the case $\hat{\mathbf{t}}_{1}=-\hat{\mathbf{t}}_{2}$ is unlikely), the circular arc will have infinite radius and the solutions for $\mathbf{P}_{2}$ and $\mathbf{P}_{3}$ are

$$
\begin{aligned}
& \mathbf{P}_{2}=\mathbf{P}_{1}+L \hat{\mathbf{t}}_{1} \\
& \mathbf{P}_{3}=\mathbf{P}_{1}+\epsilon L \hat{\mathbf{t}}_{1} .
\end{aligned}
$$

